\title{
A Kinetic Model for the Spread of Rumor in Emergencies
}

\author{
Guanghua Chen, ${ }^{1}$ Huizhang Shen, ${ }^{1}$ Teng Ye, ${ }^{1}$ Guangming Chen, ${ }^{2}$ and Naphtali Kerr ${ }^{3}$ \\ ${ }^{1}$ Antai College of Economics and Management, Shanghai Jiao Tong University, Shanghai 200052, China \\ ${ }^{2}$ School of Mechanical Engineering, Shanghai Jiao Tong University, Shanghai 200052, China \\ ${ }^{3}$ School of International Education, Shanghai Jiao Tong University, Shanghai 200052, China
}

Correspondence should be addressed to Huizhang Shen; shenhzsjtu@hotmail.com

Received 3 July 2013; Revised 1 November 2013; Accepted 11 November 2013

Academic Editor: Tetsuji Tokihiro

Copyright (C) 2013 Guanghua Chen et al. This is an open access article distributed under the Creative Commons Attribution License, which permits unrestricted use, distribution, and reproduction in any medium, provided the original work is properly cited.

\begin{abstract}
To study rumor spreading, a novel rumor spreading model with latent, constant recruitment, and varying total population is investigated. We carry out a global qualitative analysis of the rumor spreading model with latent, constant recruitment, and varying total population and studied the existence and stability of the rumor-elimination and prevailing equilibria and the impact different management strategies have on the rumor spreading process. The Runge-Kutta method is used for numerical simulation, and the simulation results imply that increasing the efforts to crack down on rumor spreaders, the timely disclosure of transparent information, the popularization of scientific knowledge, and the improvement of the ignorants' discrimination may be effective rumor control measures.
\end{abstract}

\section{Introduction}

Rumor is a typical social phenomenon that runs through the whole evolutionary history of mankind, and its spreading plays a significant role in a variety of human affairs [1-4]. A rumor is "an unverified account or explanation of events circulating from person to person and pertaining to an object, event, or issue in public concern" [2]. Rumor is usually created to defame someone or to spread false information about public events; it not only infringes upon others' interests, but also poses a threat to social stability, and it appears when a group tries to make sense of an ambiguous, uncertain, or chaotic situation. For example, in 2011, the Fukushima Daiichi nuclear disaster resulted from a 9.0-magnitude earthquake and ensuing tsunami in Japan caused a salt-buying frenzy in China. This salt-buying frenzy came from the rumors that Japan's nuclear leak would contaminate the sea off east China's Shandong province, thereby compromising the future safety of salt taken from the sea, and additionally it is believed that iodized salt helps protect people from nuclear radiation or the salt produced from contaminated seawater will be unsafe to eat. With the rumors spreading, this frenzy swept across China and caused social panic as well as instability in just a few days. From the afternoon of March 16, 2011, people in coastal areas such as Zhejiang, Jiangsu, and Shanghai, rushed out to buy up supplies, pushing up the price tenfold, before the panic spread inland. The next day, other inland areas such as Shanxi, Hubei, Anhui, Jiangxi, Guizhou, and Sichuan provinces were also caught up in the panic buying. The situation eased after the government ordered the securing of salt supplies and as experts confirmed that salt was almost useless to counter radiation. Other rumors have triggered similar responses elsewhere. Drugs stores and health food shops in Russia's Far East and British Columbia, Canada, have reported shortages of iodine pills, despite health officials insisting that potassium iodide is not antiradiation drug. The "salt" farce not only affected market order and people's lives, but also became an "international joke" that damaged the national image. The spread of rumors often results in financial loss, social instability, and, in extreme cases, even injuries and loss of lives [5]. Therefore, to study the spread of rumors under emergencies is an urgent issue for crisis management, and the cracking down on rumors is of great importance.

Rumors can be viewed as an "infection of the mind", and their spreading shows an interesting resemblance to that of epidemics. Pioneering contributions to their modeling, based on epidemic models, date back to [1,5-20]. As far as we know, Daley first proposed the similarity between epidemics and rumors using mathematical analysis [6]. A classical rumor spreading model, introduced by Daley and Kendall [7], is 
conceptually similar to the susceptible-infective-recovered (SIR) model for epidemiology. Maki and Thompson [8] focused on the analysis of the rumor spreading model based on mathematical theory and developed the MT model. Many researchers used the DK model and the MT model extensively for their quantitative studies [10-16]. Kawachi [15] presented deterministic models for the transmission of a rumor. He investigated the age-independent case and introduced four models, which are classified according to whether the population is closed or not and whether the rumor is constant or variable. Zhao and Wang [17] established an ISRW dynamical model considering the medium as a subclass to explore the mechanism of spreading of individualto-individual and medium-to-individual. Lebensztayn et al. [18] investigated the case where a new uninterested class of people exists. Z.-L. Zhang and Z.-Q. Zhang [19] studied a model for describing the interplay between rumor spreading and emergency.

In particular, recently, a susceptible-infected-removed (SIR) rumor spreading model was introduced in [20]. This model considered a closed homogeneously mixing population of $N$ individuals. The entire population is divided into three groups that are S, I, and R. Here S, I, and R stand for the people who are spreading rumor (spreaders, similar to infective), those who never heard rumor (ignorants, similar to susceptible), and the ones who heard rumor but do not spread it (stiflers, similar to removed), respectively.

In the last decades, many rumor models have been given for modeling the spread of rumor. However, the above models play a very important role.

The rumor spreading model introduced in [20] makes some assumptions about the rules of rumor spreading. For example, when an ignorant contacts a spreader, the ignorant may believe the rumor to become a spreader directly. This assumption ignores the possibility that the ignorant may experience a latent period before becoming spreader. This possibility lies in that, faced with mixed and conflicting messages, many people's judgment becomes clouded, and some ignorants have difficulty to discriminate the authenticity of information immediately after they heard the rumor. In addition, there is no inflow to the susceptible class or outflow from any of the classes in their model. Still, their model was extremely innovative and is very useful in the modeling and analysis of rumor spreading.

In order to make the rumor spreading process fit realistic cases more closely, we extend the above SIR rumor spreading model by considering the situation that when an ignorant contacts a spreader, a certain percentage of the ignorants may experience a latent period before becoming spreaders. In addition, considering the mobility of people in a certain area, we assume that there is inflow to the ignorant class and outflow from each class. Based on the above rumor spreading mechanism, we build a novel rumor spreading model called the $X Y Z R$ model to further study the dynamics of rumors spreading.

The rest of this paper is organized as follows. We describe the $X Y Z R$ rumor spreading model and discuss the existence of equilibrium in Section 2. In Section 3, we carry out a qualitative analysis of the model. Stability conditions for the rumor-elimination equilibrium and the prevailing equilibrium are derived, respectively. In Section 4, we report the numerical results and discuss the implications of these results. Finally, conclusions are drawn in Section 5.

\section{2. $X Y Z R$ Rumor Spreading Model}

We define our $X Y Z R$ rumor spreading model in the following way. Let $N(t)$ denote the total population at time $t$. We divide the population into four classes: the ignorant class, the latent class, the spreader class, and the stifler class, according to the perception and reaction of an individual to a rumor. Population densities at time $t$ are denoted by $X(t), Y(t), Z(t)$, and $R(t)$, respectively. Those who belong to the ignorant class, whom we call ignorants, do not know about the rumor. Those who belong to the latent class, whom we call latents, know about the rumor and cannot discriminate the authenticity of information immediately after he/she heard the rumor and require active effort to verify the information. The latent class also has infectious force due to the possibility that a latent who has the awareness of seeking confirmation with each other may share the information with the ignorants who are the latent's friends or relatives immediately after he/she heard the rumor; on the other hand, the latent who does not understand the rumor due to the low level of his/her scientific knowledge or forgets about the rumor is probably not going to be sharing the rumor with his/her friends or relatives. In this case, we assume that an ignorant contacts a latent with probability $\lambda \in(0,1]$. Those who belong to the spreader class, whom we call spreaders, know about the rumor and spread it actively. With the development of emergency, a spreader may spontaneously lose interest in the rumor if the rumor itself is not absorbing. In that case, we assume that an ignorant contacts one of these spreaders with probability $1-\lambda$. Those who belong to the stifler class, whom we call stiflers, know about the rumor and do not spread it. Each of which we call rumor class. By definition, we have

$$
N(t)=X(t)+Y(t)+Z(t)+R(t) .
$$

We assume that no transition of rumor class happens unless a spreader and latent contact someone, and there exists infectious force in the latent class and spreader class. The $X Y Z R$ rumor spreading model is depicted in the transfer diagram in Figure 1.

First, we consider the spread of rumor with variable population size; we assume that the rumors spread in the population with constant immigration and emigration. All recruitment is into the ignorant class and occurs at a constant $\varepsilon$, and $k_{1}, k_{2}, k_{3}$, and $k_{4}$ are the emigration rates of those in the ignorant class, the latent class, the spreader class, and the stifler class, respectively. We assume that $\varepsilon, k_{1}, k_{2}, k_{3}$, and $k_{4}$ are positive constants, and that emigration is independent of rumor class.

As shown in Figure 1, the rumor spreading rules of our $X Y Z R$ model can be summarized as follows.

(i) When an ignorant contacts a latent with probability $\lambda \in(0,1]$, the ignorant becomes a latent at a rate $\beta$, namely latent rate. 


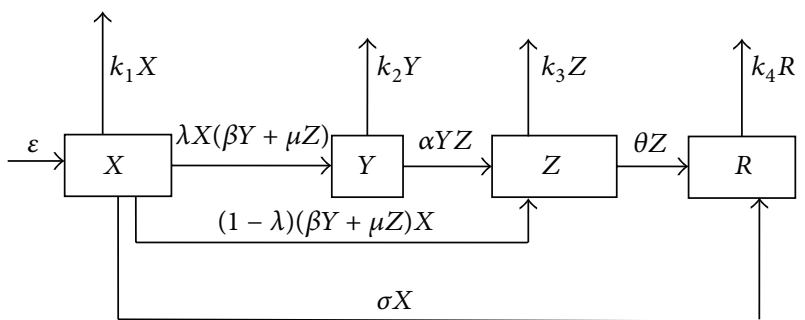

Figure 1: The flowchart of XYZR model for the rumor spreading process.

(ii) When an ignorant contacts a spreader with probability $1-\lambda$, the ignorant believes the rumor and becomes a spreader directly at a rate $\mu$, namely spreading rate.

(iii) Because some ignorants who have expertise or scientific knowledge (such as scientists or experts in the related fields) will not be affected by the rumors, hence, these ignorants directly become stiflers at a rate $\sigma$, namely stifling rate.

(iv) Any spreader may automatically lose interest in spreading, and consequently becomes a stifler at rate $\theta$, namely removed rate.

For the meantime, we assume the rumor is "constant"; that is, the same remark is transmitted at all times. We assume that $(1-\lambda)(\beta Y+\mu Z) X$ and $\lambda X(\beta Y+\mu Z)$ are ignorants that change their rumor class and become spreaders and latents, respectively. When the latents contact the spreaders, some of the latents may firmly believe the rumor and spread the rumor to the ignorants, and so, we assume that $\alpha Y Z$ latents change their rumor class and become spreaders, where $\alpha$ is a positive constant number representing the proportion of latents changing their rumor class.

The XYZR model was established based on the above assumptions according to the dynamic theory. The differential equations are described as follows:

$$
\begin{aligned}
\frac{d X}{d t} & =\varepsilon-k_{1} X-(\beta Y+\mu Z) X-\sigma X, \\
\frac{d Y}{d t} & =\lambda(\beta Y+\mu Z) X-k_{2} Y-\alpha Y Z, \\
\frac{d Z}{d t} & =(1-\lambda)(\beta Y+\mu Z) X-\left(k_{3}+\theta\right) Z+\alpha Y Z, \\
\frac{d R}{d t} & =\theta Z+\sigma X-k_{4} R, \\
\frac{d N}{d t} & =\frac{d X}{d t}+\frac{d Y}{d t}+\frac{d Z}{d t}+\frac{d R}{d t} \\
& =\varepsilon-k_{1} X-k_{2} Y-k_{3} Z-k_{4} R .
\end{aligned}
$$

From the first equation of system (2), we have that

$$
\frac{d X}{d t} \leq \varepsilon-\left(k_{1}+\sigma\right) X
$$

Therefore

$$
\limsup _{t \rightarrow \infty} X \leq \frac{\varepsilon}{k_{1}+\sigma} .
$$

From the last equation of system (2), we can see that

$$
\begin{aligned}
\frac{d N}{d t} & =\frac{d X}{d t}+\frac{d Y}{d t}+\frac{d Z}{d t}+\frac{d R}{d t} \\
& =\varepsilon-k_{1} X-k_{2} Y-k_{3} Z-k_{4} R \leq \varepsilon-\bar{k} N,
\end{aligned}
$$

where $\bar{k}=\min \left\{k_{1}, k_{2}, k_{3}, k_{4}\right\}$, thus, it can be obtained that

$$
\limsup _{t \rightarrow \infty} N \leq \frac{\varepsilon}{\bar{k}}
$$

The equation for $R$ can be omitted in our analysis as $R$ does not appear in any of the other equations. This shows that the model can be studied in the feasible region.

Consider the following:

$$
\Theta=\left\{(X, Y, Z) \in R_{+}^{3}: X \leq \frac{\varepsilon}{k_{1}+\sigma}, X+Y+Z \leq \frac{\varepsilon}{\bar{k}}\right\},
$$

where $R_{+}^{3}$ denotes the nonnegative cone and its lower dimensional faces. It can be verified that $\Theta$ is positively invariant with respect to (2).

Once the dynamics of $(X, Y, Z)$ are understood, those of $R$ can then be determined from the equation $d R / d t=\theta Z+\sigma X-$ $k_{4} R$. Then the reduced limiting dynamical system is given by

$$
\begin{aligned}
\frac{d X}{d t} & =\varepsilon-k_{1} X-(\beta Y+\mu Z) X-\sigma X \\
& =f_{1}(X, Y, Z) \\
\frac{d Y}{d t} & =\lambda(\beta Y+\mu Z) X-k_{2} Y-\alpha Y Z \\
& =f_{2}(X, Y, Z), \\
\frac{d Z}{d t} & =(1-\lambda)(\beta Y+\mu Z) X-\left(k_{3}+\theta\right) Z+\alpha Y Z \\
& =f_{3}(X, Y, Z) .
\end{aligned}
$$

We first consider the existence of equilibria of system (8).

Denote the boundary and the interior of $\Theta$ in $R_{+}^{3}$ by $\partial \Theta$ and $\stackrel{\circ}{\Theta}$, respectively. The rumor-elimination equilibrium occurs when there are no latents and spreaders in the system; that is, $Y=0$ and $Z=0$, which means that the rumors die out.

For any values of parameters, system (8) always has a rumor-elimination equilibrium $\phi_{0}=\left(\varepsilon /\left(k_{1}+\sigma\right), 0,0\right)$.

A rumor-prevailing equilibrium $\phi^{*}=\left(X^{*}, Y^{*}, Z^{*}\right)$ satisfies $X^{*}, Y^{*}, Z^{*}>0$. To find the rumor-prevailing equilibrium (or positive equilibrium) $\phi^{*}=\left(X^{*}, Y^{*}, Z^{*}\right)$, set

$$
\begin{aligned}
& f_{1}(X, Y, Z)=\varepsilon-k_{1} X-(\beta Y+\mu Z) X-\sigma X=0, \\
& f_{2}(X, Y, Z)=\lambda(\beta Y+\mu Z) X-k_{2} Y-\alpha Y Z=0, \\
& f_{3}(X, Y, Z)=(1-\lambda)(\beta Y+\mu Z) X-\left(k_{3}+\theta\right) Z+\alpha Y Z=0 .
\end{aligned}
$$


From (9), we can show that a unique $\phi^{*}$ exists with

$$
X^{*}=\frac{\left(k_{3}+\theta\right)}{\lambda k_{3} \beta+\left(k_{2}+\alpha\right) \mu+\lambda\left(\theta-k_{2} \mu\right)} \text {. }
$$

From the definition of feasible region (7), we know that $\varepsilon /\left(\left(k_{1}+\sigma\right) X^{*}\right) \geq 1$.

Define

$$
R_{0}=\frac{\varepsilon}{\left(k_{1}+\sigma\right) X^{*}}=\frac{\varepsilon\left(\lambda k_{3} \beta+\left(k_{2}+\alpha\right) \mu+\lambda\left(\theta-k_{2} \mu\right)\right)}{\left(k_{1}+\sigma\right)\left(k_{3}+\theta\right)}
$$

Then $R_{0}$ is a threshold parameter that determines the number of equilibria.

From the mathematical analysis of (9) we can see that

(1) if $R_{0} \leq 1$, then $\phi_{0}$ is the only equilibrium in $\Theta$;

(2) if $R_{0}>1$, then there are two equilibria, $\phi_{0}$ and a unique rumor-prevailing equilibrium $\phi^{*}=\left(X^{*}, Y^{*}\right.$, $\left.Z^{*}\right)$.

In the next section, we will study the property of these equilibria and perform a global qualitative analysis of model (8).

\section{Dynamical Analysis of $X Y Z R$ Rumor Spreading Model}

Theorem 1. Suppose $R_{0}<1$, the rumor-elimination equilibrium $\phi_{0}$ of system (8) is locally asymptotically stable.

Proof. The Jacobian matrix of system (8) at $\phi_{0}=\left(\varepsilon /\left(k_{1}+\right.\right.$ $\sigma), 0,0)$ is

$$
J\left(\phi_{0}\right)=\left[\begin{array}{ccc}
-k_{1}-\delta & -\beta\left(\frac{\varepsilon}{k_{1}+\sigma}\right) & -\mu\left(\frac{\varepsilon}{k_{1}+\sigma}\right) \\
0 & \lambda \beta\left(\frac{\varepsilon}{k_{1}+\sigma}\right)-k_{2} & \lambda \mu\left(\frac{\varepsilon}{k_{1}+\sigma}\right) \\
0 & (1-\lambda) \beta\left(\frac{\varepsilon}{k_{1}+\sigma}\right) & (1-\lambda) \mu\left(\frac{\varepsilon}{k_{1}+\sigma}\right)-\left(k_{3}+\theta\right)
\end{array}\right] .
$$

One eigenvalue of $J\left(\phi_{0}\right)$ is $\lambda_{1}=-\left(k_{1}+\delta\right)<0$. The other two eigenvalues $\lambda_{2}, \lambda_{3}$ are eigenvalues of the $2 \times 2$ matrix.

$$
L=\left[\begin{array}{cc}
\lambda \beta\left(\frac{\varepsilon}{k_{1}+\sigma}\right)-k_{2} & \lambda \mu\left(\frac{\varepsilon}{k_{1}+\sigma}\right) \\
(1-\lambda) \beta\left(\frac{\varepsilon}{k_{1}+\sigma}\right) & (1-\lambda) \mu\left(\frac{\varepsilon}{k_{1}+\sigma}\right)-\left(k_{3}+\theta\right)
\end{array}\right] .
$$

Then we obtain

$$
\begin{aligned}
\operatorname{tr}(L)= & \lambda \beta\left(\frac{\varepsilon}{k_{1}+\sigma}\right)-k_{2}+\left(k_{3}+\theta\right) \\
& \times\left[\frac{(1-\lambda) \mu\left(\varepsilon /\left(k_{1}+\sigma\right)\right)}{\left(k_{3}+\theta\right)}-1\right] .
\end{aligned}
$$

Using our assumption that $R_{0}<1$, we have

$$
\begin{gathered}
\lambda \beta\left(\frac{\varepsilon}{k_{1}+\sigma}\right)-k_{2}<0, \\
\frac{(1-\lambda) \mu\left(\varepsilon /\left(k_{1}+\sigma\right)\right)}{\left(k_{3}+\theta\right)}-1<0 .
\end{gathered}
$$

Thus, we get

$$
\begin{aligned}
\operatorname{tr}(L)= & \lambda \beta\left(\frac{\varepsilon}{k_{1}+\sigma}\right)-k_{2}+\left(k_{3}+\theta\right) \\
& \times\left[\frac{(1-\lambda) \mu\left(\varepsilon /\left(k_{1}+\sigma\right)\right)}{\left(k_{3}+\theta\right)}-1\right]<0 .
\end{aligned}
$$

Simple calculations show that

$$
\begin{aligned}
\operatorname{det}(L)= & {\left[\lambda \beta\left(\frac{\varepsilon}{k_{1}+\sigma}\right)-k_{2}\right] } \\
& \times\left[(1-\lambda) \mu\left(\frac{\varepsilon}{k_{1}+\sigma}\right)-\left(k_{3}+\theta\right)\right] \\
& -\lambda \mu\left(\frac{\varepsilon}{k_{1}+\sigma}\right)(1-\lambda) \beta\left(\frac{\varepsilon}{k_{1}+\sigma}\right) \\
= & \frac{\varepsilon\left(k_{2}+\alpha\right)}{\left(k_{1}+\sigma\right)}\left(1-R_{0}\right) .
\end{aligned}
$$

Therefore, $\operatorname{det}(L)>0$ if and only if $R_{0}<1$. Hence, by RouthHurwitz criterion, the rumor-elimination equilibrium $\phi_{0}$ of system (8) is locally asymptotically stable if $R_{0}<1$. This completes the proof.

Next, we focus on investigating the global stability of the rumor-elimination equilibrium and rumor-prevailing equilibrium. Firstly, we consider the global stability of the rumor-elimination equilibrium point.

Theorem 2. The rumor-elimination equilibrium $\phi_{0}$ is

(i) globally asymptotically stable in the feasible region $\Theta$ if $R_{0} \leq 1$,

(ii) unstable if $R_{0}>1$. 
Proof. To prove the global asymptotic stability of $\phi_{0}$ we use the method of Lyapunov functions. Motivated by those used in [21], we take a Lyapunov function $\psi$ of form

$$
\psi=\left(\frac{\beta}{k_{2}}+\frac{\mu \alpha}{k_{2}\left(k_{3}+\theta\right)}\right) Y+\frac{\mu}{k_{3}+\theta} Z
$$

Then

$$
\begin{aligned}
\frac{d \psi}{d t}= & \left(\frac{\beta}{k_{2}}+\frac{\mu \alpha}{k_{2}\left(k_{3}+\theta\right)}\right) \frac{d Y}{d t}+\frac{\mu}{k_{3}+\theta} \frac{d Z}{d t} \\
= & {\left[\left(\frac{\beta}{k_{2}}+\frac{\mu \alpha}{k_{2}\left(k_{3}+\theta\right)}\right)\right] } \\
& \times\left[\lambda(\beta Y+\mu Z) X-k_{2} Y-\alpha Y Z\right]+\frac{\mu}{k_{3}+\theta} \\
& \times\left[(1-\lambda)(\beta Y+\mu Z) X-\left(k_{3}+\theta\right) Z+\alpha Y Z\right] \\
= & \left(\frac{k_{1}+\delta}{\varepsilon} R_{0} X-1\right)(\beta Y+\mu Z) .
\end{aligned}
$$

$$
J\left(\phi^{*}\right)=\left[\begin{array}{ccc}
-k_{1}-\left(\beta Y^{*}+\mu Z^{*}\right)-\delta & -\beta X^{*} & -\mu X^{*} \\
\lambda\left(\beta Y^{*}+\mu Z^{*}\right) & \lambda \beta X^{*}-k_{2}-\alpha Z^{*} & \lambda \mu X^{*}-\alpha Y^{*} \\
(1-\lambda)\left(\beta Y^{*}+\mu Z^{*}\right) & (1-\lambda) \beta X^{*}+\alpha Z^{*} & (1-\lambda) \mu X^{*}-\left(k_{3}+\theta\right)+\alpha Y^{*}
\end{array}\right] .
$$

Since $R_{0} \leq 1$ and $X \leq \varepsilon /\left(k_{1}+\sigma\right)$, we have

$$
\begin{aligned}
\frac{d \psi}{d t} & =\left(\frac{k_{1}+\delta}{\varepsilon} R_{0} X-1\right)(\beta Y+\mu Z) \\
& \leq\left(R_{0}-1\right)(\beta Y+\mu Z) \leq 0 .
\end{aligned}
$$

Furthermore, $d \psi / d t=0 \Leftrightarrow Y=Z=0$ or $R_{0}=1$ and $X=$ $\bar{\Theta}$ of $\Theta$ where $d \psi / d t=0$ is the singleton $\left\{\phi_{0}\right\}$. By LaSalle's invariance principle [21], $\phi_{0}$ is globally asymptotically stable in $\Theta$, and the proof is completed.

Next, we focus on investigating the global stability of the rumor-prevailing equilibrium $\phi^{*}$ when $R_{0}>1$.

Theorem 3. If $R_{0}>1$, then the rumor-prevailing equilibrium $\phi^{*}$ of the system (8) is globally stable in $\stackrel{\circ}{\Theta}$.

Proof. By Routh-Hurwitz criterion, to prove the global stability of $\phi^{*}$, we need to prove that all eigenvalues of corresponding Jacobian matrix $J$ of system (8) have negative real parts. Then we want to show, when $R_{0}>1$, that the RouthHurwitz conditions hold, namely, $\operatorname{tr}(J)<0$, $\operatorname{det}(J)<0$, and $\operatorname{tr}(J) * M-\operatorname{det}(J)<0$, respectively.

The Jacobian matrix of system (8) at $\phi^{*}$ is $\varepsilon /\left(k_{1}+\sigma\right)$. Therefore the largest invariant set in the closure
Simple calculations show that

$$
\begin{aligned}
\operatorname{tr}\left(J\left(\phi^{*}\right)\right)= & -k_{1}-\left(\beta Y^{*}+\mu Z^{*}\right)-\delta+\lambda \beta X^{*}-k_{2}-\alpha Z^{*} \\
& +(1-\lambda) \mu X^{*}-\left(k_{3}+\theta\right)+\alpha Y^{*}<0 .
\end{aligned}
$$

Now we calculate

$$
\begin{aligned}
\operatorname{det}\left(J\left(\phi^{*}\right)\right)= & \left(-k_{1}-\delta-\beta Y^{*}-\mu Z^{*}\right) \\
& \times\left(\lambda \beta X^{*}-k_{2}-\alpha Z^{*}\right) \\
& \times\left(\mu X^{*}-\lambda \mu X^{*}-k_{3}-\theta+\alpha Y^{*}\right) \\
& +\left(-\beta X^{*}\right)\left(\lambda \mu X^{*}-\alpha Y^{*}\right) \\
& \times\left((1-\lambda)\left(\beta Y^{*}+\mu Z^{*}\right)\right) \\
& +\left(-\mu X^{*}\right)\left((1-\lambda) \beta X^{*}+\alpha Z^{*}\right) \\
& \times \lambda\left(\beta Y^{*}+\mu Z^{*}\right)<0 .
\end{aligned}
$$




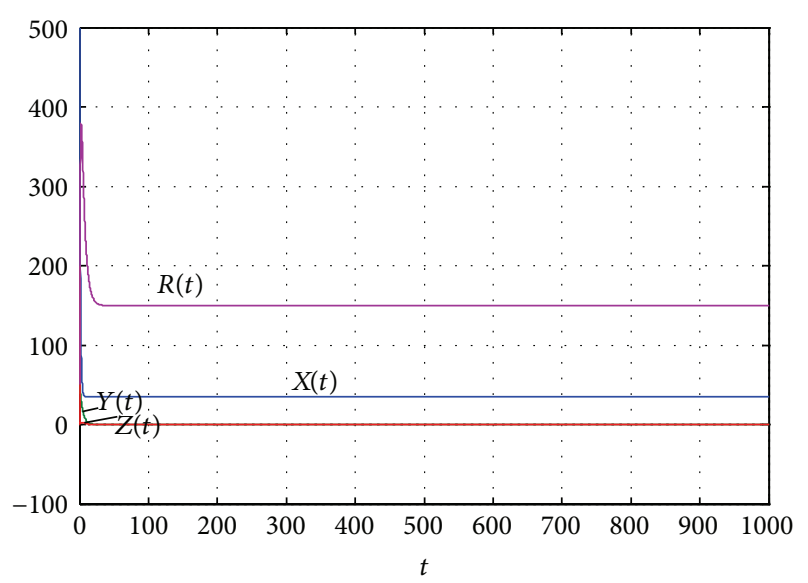

Figure 2: When $\varepsilon=3, k_{1}=0.0005, k_{2}=0.0002, k_{3}=0.04, \beta=$ $0.0005, k_{4}=0.2, \mu=0.00005, \sigma=0.85, \alpha=0.25, \theta=0.001$, $\lambda=0.06$, and $R_{0}=0.2725<1$.

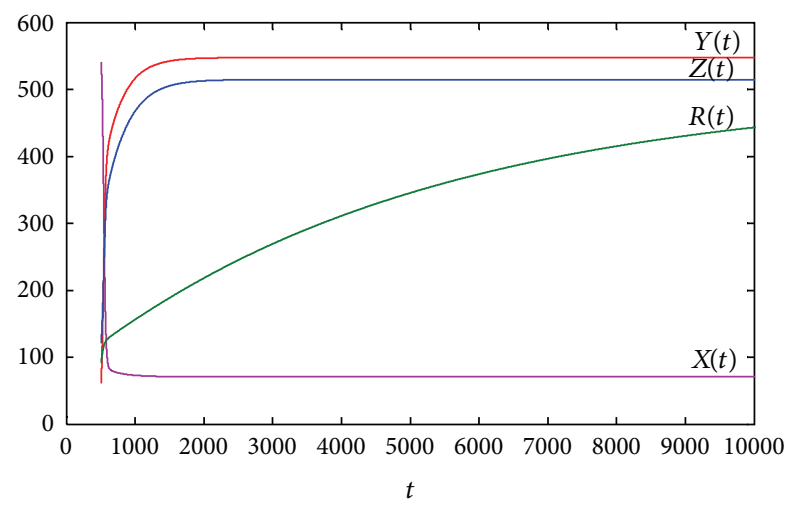

FIGURE 3: When $\varepsilon=3, k_{1}=0.00005, k_{2}=0.002, k_{3}=0.004$, $\beta=0.00015, \lambda=0.6, k_{4}=0.0002, \mu=0.00005, \sigma=0.0015$, $\alpha=0.0015, \theta=0.0001$, and $R_{0}=28.5484>1$.

\section{Discussions and Simulations}

In this section, we report the numerical results and discuss the implications of these results. We carry out numerical simulations of our model (2) by using the Runge-Kutta method and the software MATLAB 7.0. First, the obtained results of theoretical analysis (Theorems 2 and 3) are numerically validated (Figures 2 and 3 ).

Numerical results carried out for the system (2) show that the rumor "dies out" when the threshold parameter $R_{0}<1$ (Figure 2), and the rumor persists at a "prevalent" level when $R_{0}>1$ (Figure 3).

Recall the threshold parameter $R_{0}$, we have

$$
R_{0}=\frac{\varepsilon}{\left(k_{1}+\sigma\right) X^{*}}=\frac{\varepsilon\left(\lambda k_{3} \beta+\left(k_{2}+\alpha\right) \mu+\lambda\left(\theta-k_{2} \mu\right)\right)}{\left(k_{1}+\sigma\right)\left(k_{3}+\theta\right)} .
$$

It is straightforward from (26) that $R_{0}$ increases as $\mu$ or $\beta$ increases, and $R_{0}$ decreases as $\sigma$ increases. In reality, if

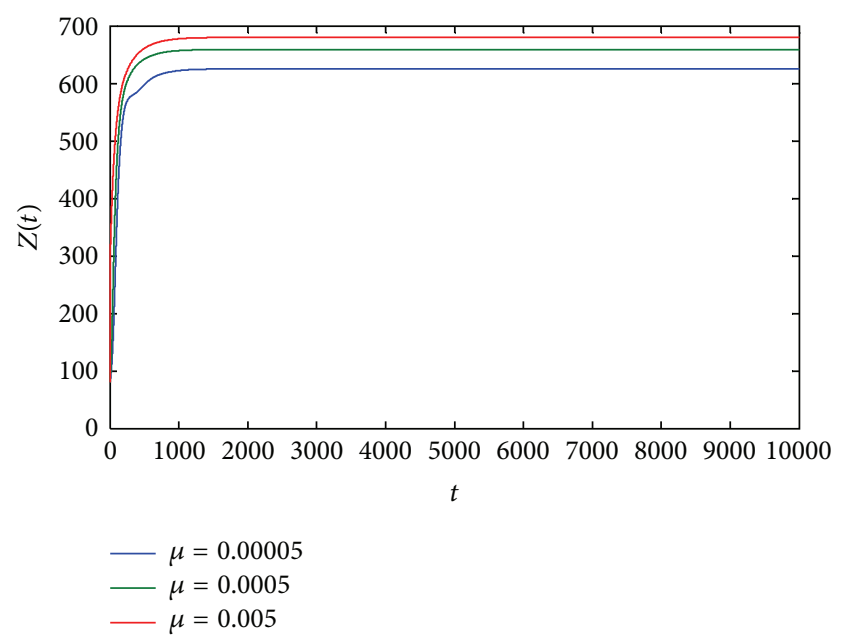

Figure 4: Size of the spreader class over time under different spreading rate $\mu$ with $\varepsilon=3, k_{1}=0.00005, k_{2}=0.002, k_{3}=0.004$, $k_{4}=0.0002, \beta=0.00015, \sigma=0.0015, \alpha=0.015, \theta=0.0001$, and $\lambda=0.6$.

there exist many latents and spreaders, which means that the parameters $\mu$ and $\beta$ will be sufficiently large such that $R_{0}>1$, then the rumors will persist at a "prevalent" level. Conversely, if the government departments made an immediate response which includes cracking down the rumor spreaders, promulgating authoritative information through authoritative channels, and improving the ignorants' discrimination, then the parameters $\mu$ and $\beta$ will become sufficiently small and $\sigma$ becomes sufficiently larger such that $R_{0}<1$, and the rumor will "die out."

Furthermore, from the perspective of crisis management strategy, it should be noted that the government departments cracking down the rumor spreaders will lead to the decrease of the spreading rate $\mu$, the timely disclosure of transparent information will result in the decrease of the latent rate $\beta$, and the popularization of scientific knowledge and the improvement of the ignorants' discrimination will lead to the increase of the stifler rate $\sigma$.

The above analysis indicates that $\mu, \beta$, and $\sigma$ that appear in the threshold parameter $R_{0}$ are three key parameters in the $X Y Z R$ rumor spreading model. Therefore, to offer useful suggestions to crisis manager, we will carry out numerical simulations by varying the above three parameters to investigate the impact corresponding management strategies have on the rumor spreading process, respectively, and discuss these simulation results with the "Chinese salt-buying frenzy case."

Figure 4 shows how the size of the spreader class changes over time for different spreading rates $\mu$ with the other parameters fixed. From Figure 4, we can see that the lower the spreading rate $\mu$, the smaller the number of rumor spreaders class. This is expected because as the spreading rate $\mu$ decreases, there is lower spreading capacity for spreaders, which means fewer chances for spreaders to be in contact with ignorants, making the spreading of a rumor much harder. Consider the Chinese salt-buying frenzy 


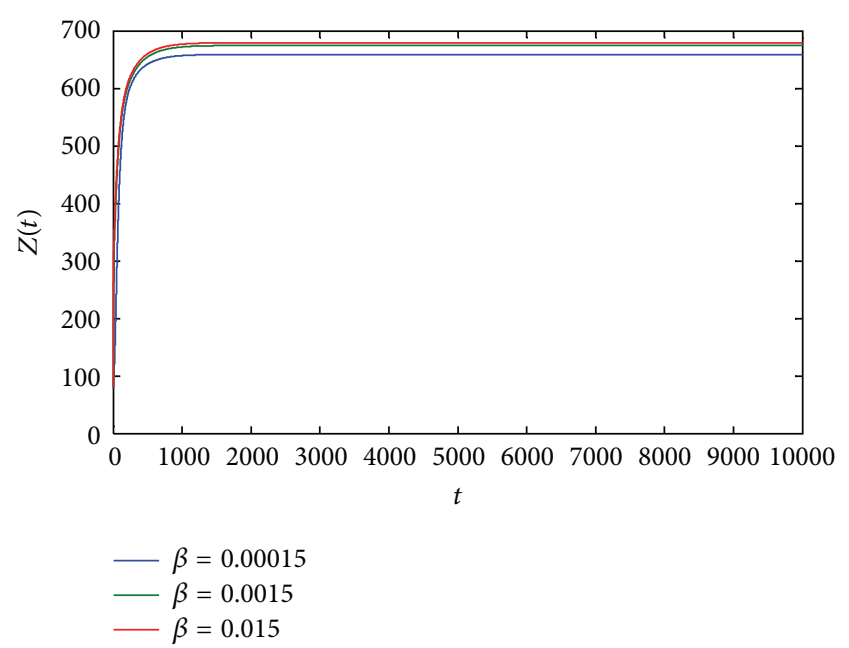

FIGURE 5: Size of the spreader class over time under different latent rate $\beta$ with $\varepsilon=3, k_{1}=0.00005, k_{2}=0.002, k_{3}=0.004, k_{4}=0.0002$, $\mu=0.00005, \sigma=0.0015, \alpha=0.015, \theta=0.0001$, and $\lambda=0.6$.

case, Chinese government and media showed a rapid and effective response in coping with the panic, such as Chinese public security authorities quickly detaining several people for allegedly fabricating and spreading online rumors about "a salt shortage" after tsunami crippled Japan's Fukushima Daiichi nuclear power plant triggered panic buying and hoarding of table salt in China, which timely controlled the source and spread of the rumors, and the salt-buying frenzy was under control effectively. One possible interpretation on this is that, in reality, if there is no strict legal punishments on those caught spreading rumors, the negative factors will run wild to destroy the social order and even pose a threat to social stability, which will bring great damage to people and society. This reflects the reality that clearing up the roots and causes of the rumors by increasing the efforts to crack down on rumor spreaders is crucial to curbing rumors.

Figure 5 describes how the size of the spreader class changes over time for different latent rate $\beta$ with the other parameters fixed. As shown in Figure 5, the lower the latent rate $\beta$, the smaller the number of rumor spreaders class. Therefore, the new added factors have affected the process of rumor spreading. With a lower $\beta$ value, fewer ignorants become the latents. Furthermore, it should be noted that some of these latents can transform to the spreaders when they believe the rumors they heard, and this process postpones the time of the spreaders' final disappearance. This implies that authorities should release information with detailed facts immediately after rumors begin to circulate, because the preciseness and openness of crisis information was helpful to dispel public panic. Consider the Chinese salt-buying frenzy case, in the initial phase of the event, faced with mixed and conflicting messages, many people's judgment becomes clouded, and people are eager to acquire authentic information through authoritative channels. Moreover, the Chinese government's transparent and active approach to releasing information regarding the nuclear leak accident in Japan should be praised. The relevant government

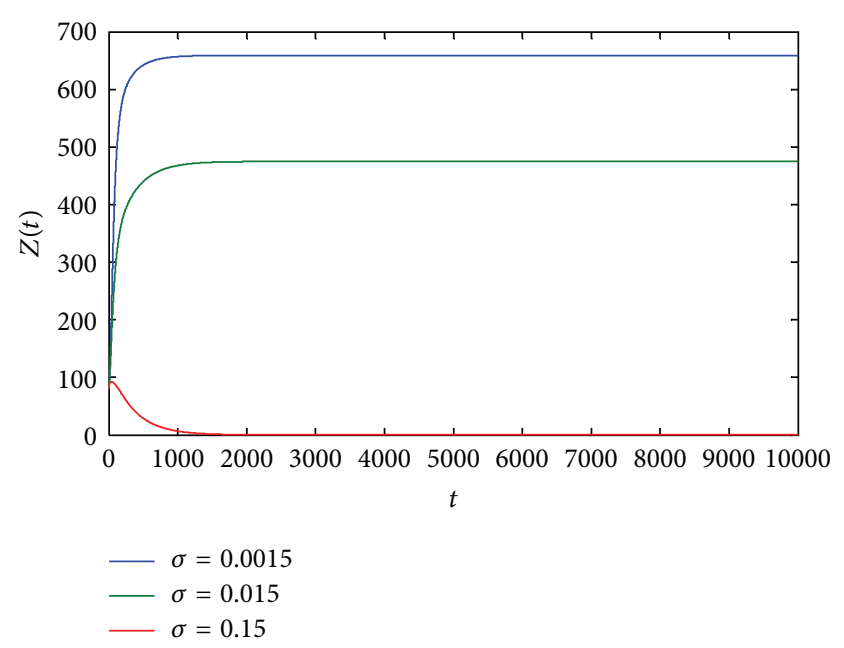

FIGURE 6: Size of the spreader class over time under different stifling rate $\sigma$ with $\varepsilon=3, k_{1}=0.00005, k_{2}=0.002, k_{3}=0.004, k_{4}=0.0002$, $\beta=0.00015, \mu=0.00005, \alpha=0.015, \theta=0.0001$, and $\lambda=0.6$.

departments immediately worked out policies to stabilize the salt market by increasing supply and strengthening supervision. This suggests that getting a timely response can minimize the hazards.

Figure 6 illustrates how the size of the spreader class changes over time for different stifling rate $\sigma$ with the other parameters fixed. From Figure 6, we can see that the higher the stifling rate $\sigma$, the smaller the peak value of the spreader class, and the higher the stifling rate is $\sigma$, the faster the rumor terminates. As shown in Figure 6, the number of spreaders shows a much greater decline while the value of $\sigma$ increases from 0.0015 to 0.15 . With a higher $\sigma$ value, more ignorants (such as experts or doctors who know the professional knowledge of nuclear protection) directly go to the stiflers status and do not participate further in the spreading dynamics, which leads to smaller number of the spreaders. It should be noted that the increase of the $\sigma$ value mainly depends on the popularization of scientific knowledge and the improvement of the ignorants' discrimination. Moreover, the average level of people's scientific knowledge is, to some degree, very low, which leads to easily believing in rumors. Only 3.27 percent of Chinese population has basic scientific knowledge, according to a survey conducted by the China Science and Technology Museum in 2010. This reflects the reality that the popularization of scientific knowledge is needed, and related experts and departments should undertake due obligations. The results coincide with the fact of the evolution of hoarding iodized salt. Consider the Chinese salt-buying frenzy case, due to a few people's lack of basic scientific knowledge about radiation, they rushed to buy iodized salt in panic after the nuclear leak in Japan. However, it is worth mentioning that Chinese government and media showed a rapid and effective response in coping with the panic. Besides the timely and transparent release of the monitoring results, the country's media outlets also began to spread scientific knowledge about radiation, such as its definition and impact on people's health. State-owned China Central Television kept inviting 
specialists almost every day to explain the facts about nuclear power to the public. After the authorities announced that the rumor was absurd and malicious then the rumor perished rapidly. It is worth mentioning that this time the panic buying salt relay panic buying of Radix Isatidis in China, signifying the popularization of scientific knowledge of shoulder heavy responsibilities.

\section{Conclusion}

In this paper, we considered some more realistic factors and extended the SIR rumor spreading model in [20] to make it closer to real life. Our stability analysis approach is quite different from that adopted in [20]. We have carried out a global qualitative analysis of a $X Y Z R$ rumor spreading model with latent and constant recruitment and varying total population and studied the existence and stability of the rumor-elimination and prevailing equilibria and the impact different management strategies have on the rumor spreading process.

We derived that the dynamics of system (2) are completely determined by the threshold parameter $R_{0}$. In terms of the threshold parameter $R_{0}$, our main results indicate that when $R_{0}<1$, the rumor-elimination equilibrium $\phi_{0}$ is globally asymptotically stable in the feasible region $\Theta$, and thus that the spread of rumor will die out spontaneously (Figure 2). When $R_{0}>1$, the rumor-prevailing equilibrium $\phi^{*}$ exists and is globally stable in $\stackrel{\circ}{\Theta}$, so that the rumor, if initially present, will persist at the unique prevailing equilibrium level (Figure 3).

From the perspective of crisis management, the numerical results imply that increasing the efforts to crack down on rumor spreaders, the timely disclosure of transparent information and the popularization of scientific knowledge and the improvement of the ignorants' discrimination may be effective rumor control measures.

\section{Acknowledgments}

The authors are grateful for the well-thought-out suggestions and comments from the anonymous reviewers and the editors of this journal. This study was supported by Grants from the Major Program of China National Social Science Fund (Grant no. 11\&ZD174), China National Natural Science Foundation (Grant no. 71071096), and Research Fund for the Doctoral Program of Higher Education of China (Grant no. 20070248054).

\section{References}

[1] L. Zhao, Q. Wang, J. Cheng, Y. Chen, J. Wang, and W. Huang, "Rumor spreading model with consideration of forgetting mechanism: a case of online blogging LiveJournal," Physica A, vol. 390, no. 13, pp. 2619-2625, 2011.

[2] W. A. Peterson and N. P. Gist, "Rumor and public opinion," American Journal of Sociology, vol. 57, no. 2, pp. 159-167, 1951.
[3] P. Bordia and N. DiFonzo, "When social psychology became less social: prasad and the history of rumor research," Asian Journal of Social Psychology, vol. 5, no. 1, pp. 49-61, 2002.

[4] G. A. Fine and P. A. Turner, Whispers on the Color Line: Rumor and Race in America, University of California Press, Berkeley, Calif, USA, 2001.

[5] L. J. Zhao, J. J. Wang, Y. C. Chen, Q. Wang, J. J. Cheng, and H. Cui, "SIHR rumor spreading model in social networks," Physica A, vol. 391, no. 7, pp. 2444-2453, 2012.

[6] D. J. Daley and D. G. Kendall, "Epidemics and rumours," Nature, vol. 204, no. 4963, p. 1118, 1964.

[7] D. J. Daley and D. G. Kendall, "Stochastic rumours," Journal of the Institute of Mathematics and its Applications, vol. 1, pp. 4255, 1965.

[8] D. P. Maki and M. Thompson, Mathematical Models and Applications, Prentice-Hall, Englewood Cliffs, NJ, USA, 1973.

[9] D. J. Daley, J. Gani et al., Epidemic Modelling, Cambridge University Press, Cambridge, UK, 2000.

[10] S. Belen, E. Kropa, and G. W. Weber, "Rumours within time dependent Maki-Thompson Model,” Working Paper, 2008.

[11] W. Huang, "On rumour spreading with skepticism and denia," Working Paper, 2011.

[12] C. Lefèvre and P. Picard, "Distribution of the final extent of a rumour process," Journal of Applied Probability, vol. 31, no. 1, pp. 244-249, 1994.

[13] B. Pittel, "On a Daley-Kendall model of random rumours," Journal of Applied Probability, vol. 27, no. 1, pp. 14-27, 1990.

[14] K. Thompson, R. Castro Estrada, D. Daugherty, and A. Cintrn Arias, "A deterministic approach to the spread of rumors," Working Paper, Washington, DC, USA, 2003.

[15] K. Kawachi, "Deterministic models for rumor transmission," Nonlinear Analysis. Real World Applications, vol. 9, no. 5, pp. 1989-2028, 2008.

[16] K. Kawachi, M. Seki, H. Yoshida, Y. Otake, K. Warashina, and H. Ueda, "A rumor transmission model with various contact interactions," Journal of Theoretical Biology, vol. 253, no. 1, pp. 55-60, 2008.

[17] X. X. Zhao and J. Z. Wang, "Dynamical model about rumor spreading with medium," Discrete Dynamics in Nature and Society, vol. 2013, Article ID 586867, 9 pages, 2013.

[18] E. Lebensztayn, F. P. Machado, and P. M. Rodríguez, "Limit theorems for a general stochastic rumour model," SIAM Journal on Applied Mathematics, vol. 71, no. 4, pp. 1476-1486, 2011.

[19] Z.-L. Zhang and Z.-Q. Zhang, "An interplay model for rumour spreading and emergency development," Physica A, vol. 388, no. 19, pp. 4159-4166, 2009.

[20] L. J. Zhao, H. X. Cui, X. Y. Qiu, X. L. Wang, and J. J. Wang, "SIR rumor spreading model in the new media age," Physica A, vol. 392, no. 4, pp. 995-1003, 2013.

[21] Z. E. Ma, Y. C. Zhou, and J. H. Wu, Modeling and Dynamics of Infectious Diseases, High Education Press \& World Scientific, 2009. 


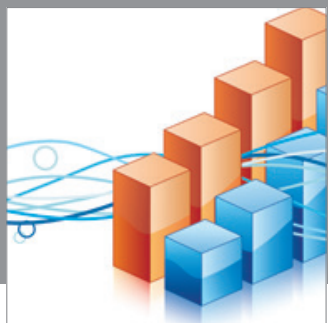

Advances in

Operations Research

mansans

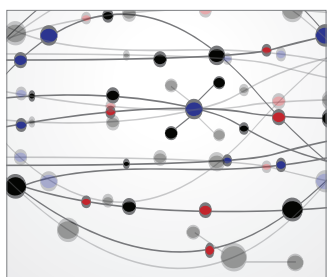

The Scientific World Journal
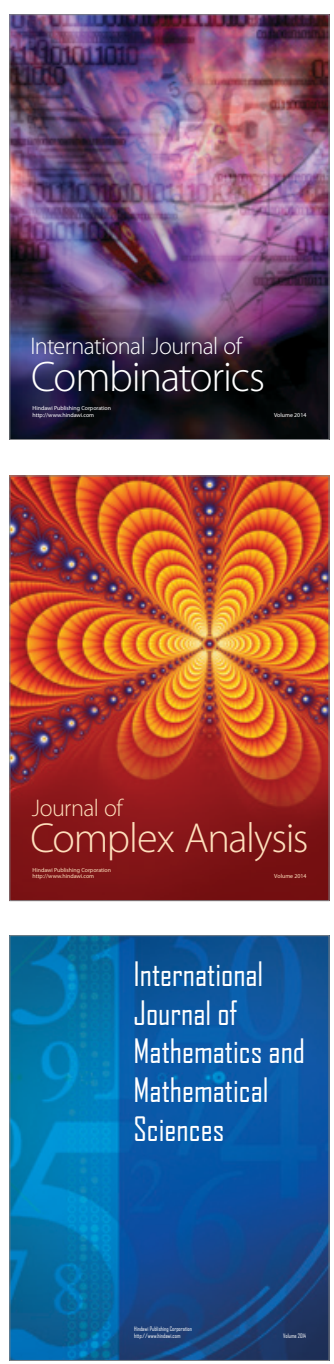
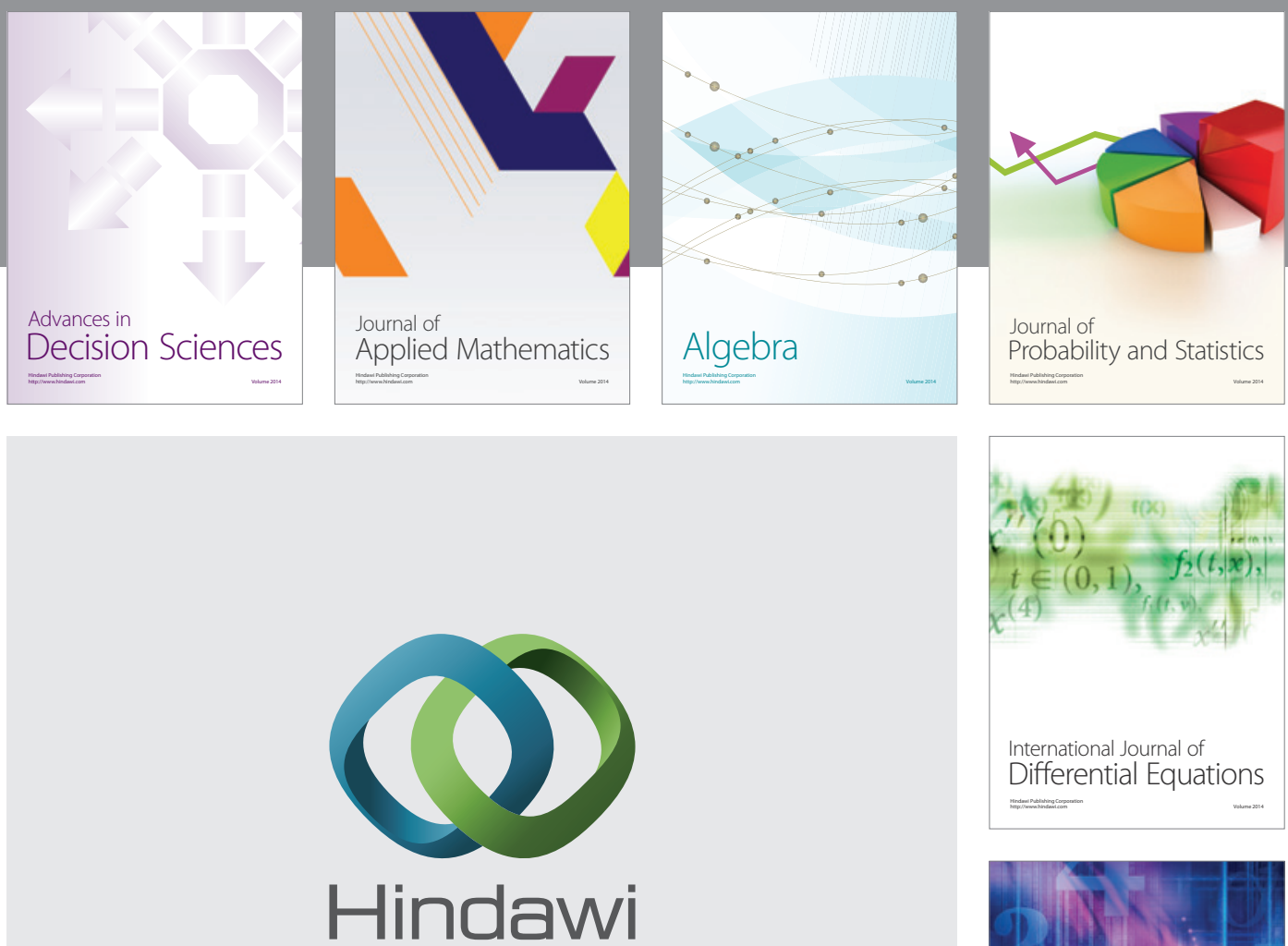

Submit your manuscripts at http://www.hindawi.com
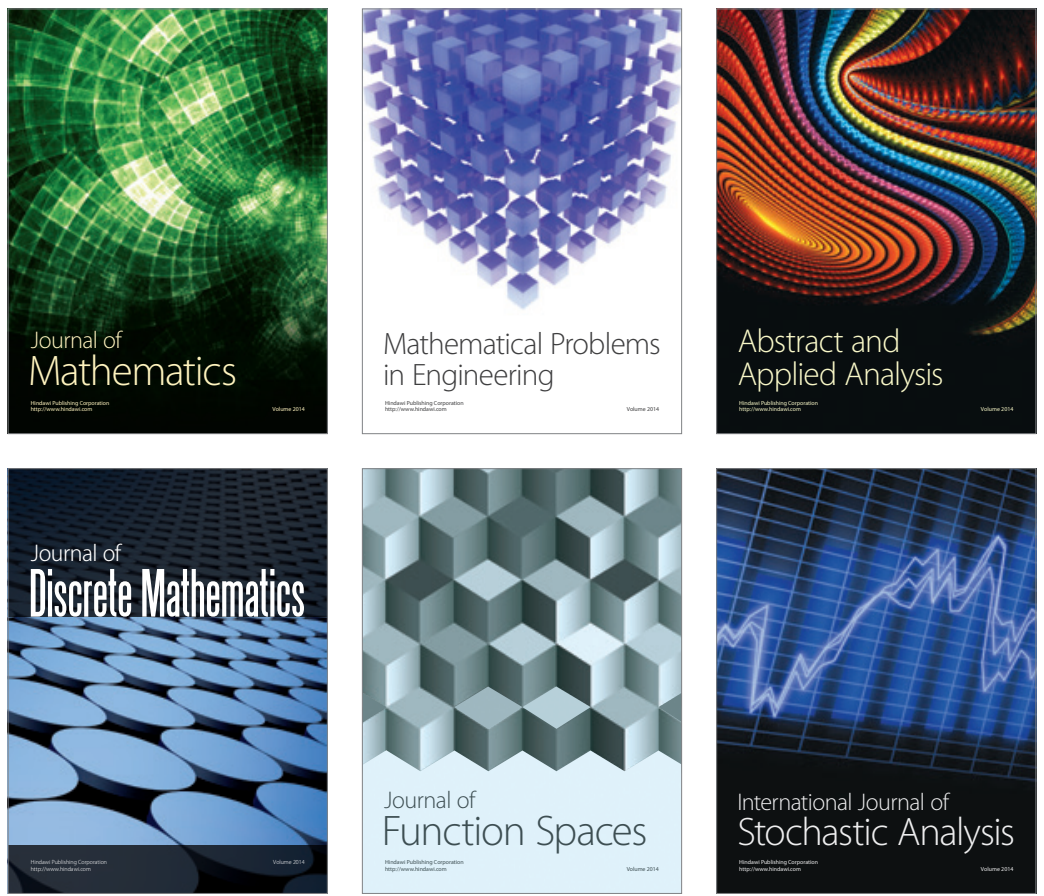

Journal of

Function Spaces

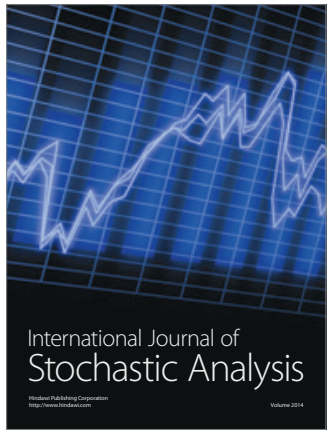

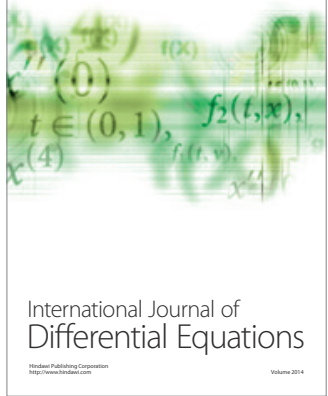
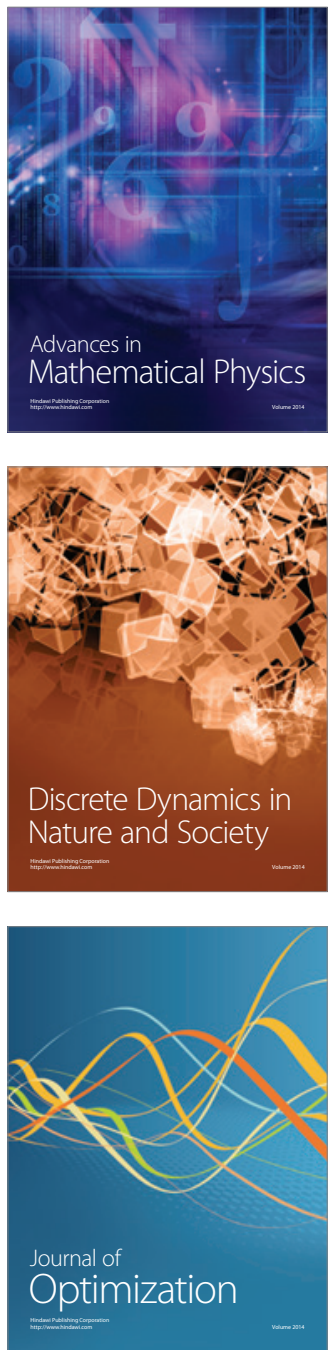